\title{
Association analysis of CYP2A6 polymorphism to sudden and unexpected death of infants
}

\author{
Motoki Osawa $^{1 *}$, Yoshihiko Inaoka ${ }^{1}$, Masato Nakatome ${ }^{2}$, Keiko Miyashita ${ }^{1}$, Eriko Ochiai ${ }^{1}$, Yu Kakimoto ${ }^{1}$, Fumiko Satoh ${ }^{1}$ and Ryoji Matoba $^{3}$ \\ ${ }^{1}$ Department of Forensic Medicine, Tokai University School of Medicine, Isehara, Kanagawa 259-1193, Japan \\ ${ }^{2}$ Department of Legal Medicine, Fujita Health University School of Medicine, Aichi, Japan \\ ${ }^{3}$ Legal Medicine, Department of Social and Environmental Medicine, Division of Preventive and Environmental Medicine, Osaka University Graduate School of \\ Medicine, Osaka, Japan
}

\begin{abstract}
Exposure to tobacco smoke is the major risk factor for the occurrence of sudden and unexpected death in infants. The component of nicotine, which is metabolized mainly by cytochrome P450 (CYP) 2A6, is of particular concern because of its adverse effects on fetal development. In this study, we performed an association study of CYP2A6 polymorphism with SIDS subjects. Genotyping of CYP2A6 was performed using PCR-RFLP method. The subjects were consisted of suspected SIDS subjects $(n=71)$ at autopsy and healthy Japanese adults $(n=225)$ for the control. Major allele frequencies of ${ }^{*} 1 A$, ${ }^{*} 1 B$, and $* A$ were $0.436,0.364$, and 0.200 , respectively, in the control group under Hardy-Weinberg equilibrium $\left(x^{2}=1.66\right)$. Relative to the frequency of $C Y P 2 A 6^{*} 1 A$, the odds ratio and $95 \%$ confidence intervals of ${ }^{*} 1 B$ and $* A$ in the SIDS group were $1.20(0.79-1.80)$ and $0.71(0.41-1.25)$, respectively. In homozygote-based analysis to ${ }^{*} 1 A /{ }^{*} 1 A$, the odds ratio and $95 \%$ confidence intervals of ${ }^{*} 1 B /{ }^{*} 1 B$ and $* 4 A / * 4 A$ in healthy controls and SIDS samples were $1.50(0.69-3.24)$ and $0.55(0.11-2.78)$, respectively. The results of this study suggest that polymorphism of $C Y P 2 A 6$ plays no role in increased susceptibility of sudden infant death from unknown causes.
\end{abstract}

\section{Introduction}

Sudden infant death syndrome (SIDS) has been reduced through the public health efforts, particularly through the 'Back to Sleep' campaign, but it still constitutes a major cause of death during the early neonatal period. Exposure to tobacco smoke is the major risk factor for the occurrence of SIDS because of its effects on the developing nervous system in the fetus and during the postnatal period [1]. A number of epidemiological studies have demonstrated that statistically significant increases of SIDS, such as a 2 to 3 -fold relative risk, have been attributed to maternal smoking [2,3]. A variety of components are present in the smoke, but nicotine is of particular concern in relation to SIDS because of its penetrance in the placenta and its adverse effects on fetal development.

The majority $(80-90 \%)$ of intake nicotine is metabolized to the inactive forms of cotinine and 3'-hyroxycotinine by cytochrome P450 (CYP) 2A6 in the liver [4]. A large interindividual difference in the metabolism is evident, which is mainly attributed to CYP2A6 polymorphism. Three major alleles of ${ }^{\star} 1 A,{ }^{\star} 1 B$ and ${ }^{\star} 4 A$, are present in eastern Asia populations $[5,6]$. In addition to the wild type of ${ }^{\star} 1 A,{ }^{\star} 1 B$ has a gene conversion of a part of the $3^{\prime}$ flanking region with $C Y P 2 A 7$, which gives rise to a limited effect of enzymatic activity [7]. In contrast, $C Y P 2 A 6^{*} 4 A$, generated by an unequal crossover event between the 3-prime flanking region of the CYP2A6 and CYP2A7 genes, is the null allele lacking the oxidase activity [8]. The presence of the various alleles clearly affects the enzymatic activity toward drugs. In particular, the ${ }^{*} 4 \mathrm{~A}$ homozygote exhibits almost no metabolism of nicotine in human liver microsomes. The present study was undertaken to clarify whether CYP2A6 genetic polymorphism, including the gene deletion of ${ }^{*} 4 \mathrm{~A}$ homozygote, could be related to individual susceptibility to SIDS or not.

\section{Materials and methods}

\section{Subjects}

During 2005 to 2012, DNA of 71 subjects less than 12 months old, for whom SIDS had been suspected after full autopsy and subsequent examinations, was extracted from blood obtained at autopsy held in the two forensic departments, Tokai University School of Medicine and Osaka University Graduate School of Medicine. For the control, 225 samples of DNA from healthy adult Japanese individuals who had no relatedness were used. Gender was adjusted between the two groups. This project has been approved by the institutional ethical committee of Tokai University School of Medicine. Written informed consent was obtained from healthy volunteers and parents of the suspected SIDS victims.

\section{Genotyping}

To determine the genotype, restriction fragment length polymorphism (RFLP) of PCR products using AccII and Eco81I was employed as described by Nakajima et al. [9]. The allele designation was referred from the human cytochrome P450 (CYP) allele nomenclature database (http://www.cypalleles.ki.se/). The Hardy-

Correspondence to: Motoki Osawa, M.D., Ph.D, Department of Forensic Medicine, Tokai University School of Medicine, Shimokasuya 143, Isehara, Kanagawa 259-1193, Japan, Tel: +81-463-93-1121; Fax: +81-463-92-0284; E-mail: osawa@is.icc.u-tokai.ac.jp

Key words: SIDS, CYP2A6 polymorphism, association study, nicotine

Received: March 08, 2015; Accepted: March 19, 2015; Published: March 23 2015 
Weinberg equilibrium for each allele among the controls was evaluated using the chi-square test. Genotype and allele frequencies of CYP2A6 were compared between cases and controls using the chi-square test or Fisher's exact test, and the odds ratio (OR) and 95\% confidence intervals $(95 \% \mathrm{CI})$ were calculated to evaluate the effects of differences for both allelic and genotypic distributions. Genotype association tests were performed in a case-control pattern assuming codominant (homozygote and heterozygote comparison) and dominant genetic models [10]. Differences in comparison were examined in $t$-test and Mann-Whitney test.

\section{Results}

The suspected SIDS group included 71 infants (40 male, $56.3 \%$; 31 female, $43.7 \%$ ) with mean age and S.D. of $20.2 \pm 10.9$ weeks. Details are summarized in Table 1 . In a comparison between smokers and non-smokers, younger smoking mothers with shorter gestational duration tended to suffer from infant death, but the differences were not significant (Table 2).

The CYP2A6 genotype was ascertained using the PCR-RFLP method. For the control group $(n=225)$, allele frequencies of ${ }^{\star} 1 A,{ }^{\star} 1 B$, and ${ }^{\star} 4 A$, respectively, were $0.436,0.364$, and 0.200 . The distribution of CYP2A6 genotypes among controls was not different from that expected from the Hardy-Weinberg equilibrium $\left(x^{2}=1.66, P=0.89\right)$.

Table 3 indicates the allelic and genotypic differences between the

Table 1. Baseline characteristics of $C Y P 2 A 6$ polymorphism association study subjects.

\begin{tabular}{|l|c|}
\hline Characteristics & SIDS suspected subjects $(\boldsymbol{n}=\mathbf{7 1})$ \\
\hline Infant age (weeks) & $20.2 \pm 10.9$ \\
\hline Infant sex & male, $40(56.3 \%) ;$ female, $31(43.7 \%)$ \\
\hline Weight at birth $(\mathrm{g})$ & $2864 \pm 513$ \\
\hline Gestational weeks & $38.2 \pm 2.3$ \\
\hline Prone position at scene & $35 / 62 *(56.5 \%)$ \\
\hline Mother's age & $27.5 \pm 5.4$ \\
\hline Maternal smoking & $12 / 49 *(24.5 \%)$ \\
\hline
\end{tabular}

*Information was not available for all subjects.

Table 2. Comparison of the baseline characteristics between smokers and non-smokers of the mothers.

\begin{tabular}{|l|c|c|c|}
\hline & Non-smokers $(\boldsymbol{n}=\mathbf{3 7})$ & Smokers $(\boldsymbol{n = 1 2})$ & $\boldsymbol{P}$ in $\boldsymbol{t}$-test \\
\hline Infant age (weeks) & $19.5 \pm 11.4$ & $20.8 \pm 12.6$ & 0.37 \\
\hline Weight at birth (g) & $2931 \pm 439$ & $2702 \pm 618$ & 0.12 \\
\hline Gestational weeks & $38.6 \pm 1.3$ & $37.3 \pm 3.4$ & 0.097 \\
\hline Mother's age (years) & $27.7 \pm 5.6$ & $25.4 \pm 5.8$ & 0.14 \\
\hline
\end{tabular}

Table 3. Association between genotypes of CYP $2 A 6$ and occurrence of SIDS.

\begin{tabular}{|c|c|c|c|c|}
\hline Genotype & Control $(\boldsymbol{n}=\mathbf{2 2 5})$ & SIDS $(\boldsymbol{n}=\mathbf{7 1})$ & OR & $\mathbf{9 5 \% C I}$ \\
\hline$* I A / * I A$ & $47(0.21)$ & $19(0.27)$ & 1.00 & \\
\hline$* I A / * I B$ & $64(0.28)$ & $14(0.20)$ & 0.54 & $0.25-1.19$ \\
\hline$* I A / * 4 A$ & $38(0.17)$ & $9(0.13)$ & 0.59 & $0.24-1.44$ \\
\hline$* I B / * I B$ & $33(0.15)$ & $20(0.28)$ & 1.50 & $0.69-3.24$ \\
\hline$* I B / * 4 A$ & $34(0.15)$ & $7(0.10)$ & 0.51 & $0.19-1.35$ \\
\hline$* 4 A / * 4 A$ & $9(0.04)$ & $2(0.03)$ & 0.55 & $0.11-2.78$ \\
\hline$* 4 A$ dominant & & & & \\
\hline$* I A / * 4 A, * I B / * 4 A, * 4 A / * 4 A$ & $81(0.36)$ & $18(0.25)$ & 1.00 & \\
\hline Others & $144(0.64)$ & $53(0.75)$ & 1.66 & $0.91-3.02$ \\
\hline Allele & & & & \\
\hline$* I A$ & $196(0.44)$ & $61(0.43)$ & 1.00 & \\
\hline$* I B$ & $164(0.36)$ & $61(0.43)$ & 1.20 & $0.79-1.80$ \\
\hline$* 4 A$ & $90(0.20)$ & $20(0.14)$ & 0.71 & $0.41-1.25$ \\
\hline
\end{tabular}

SIDS and control groups. When the OR for the allele frequency of the $C Y P 2 A 6^{\star} 1 A$ was defined as 1.00 , the ORs and $95 \%$ CI of $C Y P 2 A 6^{\star} 1 B$ and $C Y P 2 A 6^{*} 4 A$ in healthy controls and SIDS samples were $1.20(0.79$ $1.80)$ and $0.71(0.41-1.25)$, respectively. In the genotypic-base analysis, the respective ORs and $95 \%$ CIs of ${ }^{\star} 1 A /{ }^{\star} 1 B,{ }^{\star} 1 A /{ }^{\star} 4 A,{ }^{\star} 1 B /{ }^{\star} 1 B,{ }^{\star} 1 B /{ }^{\star} 4 A$, and ${ }^{*} 4 A /{ }^{*} 4 A$ to ${ }^{*} 1 A /{ }^{*} 1 A$ in healthy controls and SIDS samples were 0.54 (0.25-1.19), 0.59 (0.24-1.44), 1.50 (0.69-3.24), 0.51 (0.19-1.35), and 0.55 (0.11-2.78), respectively. In no dominant genetic model was any significant difference found. These results suggest that CYP2A6 genetic polymorphism is not a significant determinant affecting SIDS.

\section{Discussion}

The cause of genuine SIDS remains unknown, but the sudden and unexpected death of an infant (SUDI) is a multi-factorial event from extrinsic asphyxia to natural disease. SIDS must to be excluded from SUDI cases as possible [11]. Single-gene disorders such as QT elongation [12], fatty acid metabolism deficiency [13] and congenital central hypoventilation syndrome [14] are the potential causes. In another aspect, polygenic factors associated with central nervous system pathways are also thought to contribute to the occurrence $[15,16]$. As the main neurotoxic constituent of tobacco smoke, nicotine influences apnea through effects on nicotinic acetylcholine receptors in brainstem nuclei that control respiration and arousal [17].

The major metabolic pathway of nicotine is $C$-oxidation to cotinine. In the CYP $2 A 6^{\star} 4 A$ homozygote, the cotinine/nicotine ratio of the plasma concentration is zero at the nicotine half life of $2 \mathrm{~h}$ in the circulation [18]. This result means that the major gene responsible for nicotine metabolism is $C Y P 2 A 6$, and that ${ }^{*} 4 A$ homozygote exhibits critically impaired metabolism. Moreover, because the null allele of ${ }^{\star} 4 \mathrm{~A}$ is widely distributed at allele frequencies of 0.1 to 0.2 in eastern Asia population groups [6], we inferred that the effect of the CYP2A6 polymorphism can be evaluated clearly in the Japanese population. Although we collected as many DNA specimens as possible, no significant relation with infant sudden deaths from unknown causes was observed in the present association study.

Maternal smoking was confirmed in 12 cases among the present SIDS group from questionnaire of the status during pregnancy and after birth. Moreover, two complete deficient subjects of $C Y P 2 A 6^{*} 4 A$ homozygote were confirmed from the genotyping, but both mothers were non-smokers. We realized the uneasy collection of smoking parent cases. Another designed study might be desirable to clarify the effects of interindividual differences in nicotine metabolism.

Several groups have reported association studies of SIDS with polymorphisms in nicotine metabolism-related genes. Rand et al. [19] demonstrated that no association with SIDS was observed for genotype distribution or allele frequencies at any of three GSTT1 and CYP1A1 polymorphisms. However, Wang et al. [20] reported that polymorphisms of these genes were associated with low birth weight, suggesting susceptibility to other health problems accompanying tobacco smoke exposure. Poetsch et al. [21] showed that in G472A (E158K) of $F M O 3$, the A/A homozygote occurred more frequently in SIDS cases than in a control group, suggesting that the polymorphism G472A of FMO3 potentially acts as a genetic risk factor for SIDS. Aside from extensive studies to various genes associated with serotonin transporter and early development of autonomic nervous system, no critical determinant has been identified $[15,21,22]$. In addition to the association studies of the single gene, a massive DNA sequence analysis using the next generation sequencer or whole genome SNP analysis using microarray technique will be necessary in the future [23]. 
In conclusion, epidemiologic analysis shows that smoking is the major risk factor for the incidental occurrence of SIDS. The present genetic study of the CYP2A6 polymorphism in SIDS suspected subjects at forensic autopsy in the Japanese population indicated no significant association. However, to evaluate the effects of interindividual differences in nicotine metabolism, another strategy might be necessary because of the difficulty in collecting cases involving smoking parents.

\section{Acknowledgments}

This work was supported by a Grant-in-Aid for Scientific Research from the Ministry of Education, Culture, Sports, Science and Technology of Japan.

\section{References}

1. Blood-Siegfried J, Rende EK (2010) The long-term effects of prenatal nicotine exposure on neurologic development. J Midwifery Womens Health 55: 143-52. [Crossref]

2. Mitchell EA, Milerad J (2006) Smoking and the sudden infant death syndrome. Rev Environ Health 21:81-103. [Crossref]

3. Einarson A, Riordan S (2009) Smoking in pregnancy and lactation: a review of risks and cessation strategies. Eur J Clin Pharmacol 65: 325-30. [Crossref]

4. Nakajima M, Yamamoto T, Nunoya K, Yokoi T, Nagashima K, et al. (1996) Role of human cytochrome P4502A6 in C-oxidation of nicotine. Drug Metab Dispos 24: 1212 7. [Crossref]

5. Oscarson M, McLellan RA, Gullstén H, Yue QY, Lang MA, et al. (1999) Characterisation and PCR-based detection of a CYP2A6 gene deletion found at a high frequency in a Chinese population. FEBS Lett 448: 105-10. [Crossref]

6. Nakajima M, Kuroiwa Y, Yokoi T (2002) Interindividual differences in nicotine metabolism and genetic polymorphisms polymorphisms of human CYP2A6. Drug Metab Rev 34: 865-77. [Crossref]

7. Oscarson M, McLellan RA, Gullsten H, Agundez JAG, Benitez J, et al. (1999) Identification and characterization of novel polymorphisms in the CYP2A locus implications for nicotine metabolism. FEBS Lett 460: 321-7. [Crossref]

8. Nunoya KI, Yokoi T, Kimura K, Kainuma T, Satoh K, et al. (1999) A new CYP2A6 gene deletion responsible for the in vivo polymorphic metabolism of (+)-cis-3,5dimethyl-2-(3-pyridyl)thiazolidin-4-one hydrochloride in humans. J Pharmacol Exp Ther 289: 437-42. [Crossref]

9. Nakajima M, Yoshida R, Fukami T, McLeod HL, Yokoi T (2004) Novel human CYP2A6 alleles confound gene deletion analysis. FEBS Lett 569: 75-81. [Crossref]
10. Osawa M, Kimura R, Hasegawa I, Mukasa N, Satoh F (2009) SNP association and sequence analysis of the NOS1AP gene in SIDS. Leg Med 1: S307-8. [Crossref]

11. Krous HF, Beckwith JB, Byard RW, Rognum TO, Bajanowski T, et al. (2004) Sudden infant death syndrome and unclassified sudden infant deaths: a definitional and diagnostic approach. Pediatrics 114: 234-238. [Crossref]

12. Arnestad M, Crotti L, Rognum TO, Insolia R, Pedrazzini M, et al. (2007) Prevalence of long-QT syndrome gene variants in sudden infant death syndrome. Circulation 115 : 361-7. [Crossref]

13. Opdal SH, Rognum TO (2011) Gene variants predisposing to SIDS: current knowledge. Forensic Sci Med Pathol 7: 26-36. [Crossref]

14. Horiuchi H, Sasaki A, Osawa M, Kijima K, Ino Y, et al. (2005) Sensitive detection of polyalanine expansions in $P H O X 2 B$ by PCR using bisulfite-converted DNA. J Mol Diagn 7: 638-40. [Crossref]

15. Weese-Mayer DE, Ackerman MJ, Marazita ML, Berry-Kravis EM (2007) Sudden Infant Death Syndrome: review of implicated genetic factors. Am J Med Genet A 143A: 771-88. [Crossref]

16. Van Norstrand DW, Ackerman MJ (2010) Genomic risk factors in sudden infant death syndrome. Genome Med 2: 86. [Crossref]

17. Machaalani R, Say M, Waters KA (2011) Effects of cigarette smoke exposure on nicotinic acetylcholine receptor subunits $\alpha 7$ and $\beta 2$ in the sudden infant death syndrome (SIDS) brainstem. Toxicol Appl Pharmacol 257: 396-404. [Crossref]

18. Nakajima M, Kwon J, Zenta T, Tanaka N, Yamamoto Y, et al. (2001) Relationship between interindividual differences in nicotine metabolism and CYP2A6 genetic polymorphism in humans. Clin Pharmacol Ther 69: 72-8. [Crossref]

19. Rand CM, Weese-Mayer DE, Maher BS, Zhou L, Marazita ML, et al. (2006) Nicotine metabolizing genes GSTT1 and CYP1A1 in sudden infant death syndrome. Am J Med Genet Part A 140A: 1447-52. [Crossref]

20. Wang X, Zuckerman B, Pearson C, Kaufman G, Chen C, et al. (2002) Maternal cigarette smoking, metabolic gene polymorphism, and infant birth weight. JAMA 287: 195-202. [Crossref]

21. Poetsch M, Czerwinski M, Wingenfeld L, Vennemann M, Bajanowski T (2010) A common FMO3 polymorphism may amplify the effect of nicotine exposure in sudden infant death syndrome (SIDS). Int J Legal Med 124: 301-6. [Crossref]

22. Morley ME, Rand CM, Berry-Kravis EM, Zhou L, Fan W, et al. (2008) Genetic variation in the HTR1A gene and sudden infant death syndrome. Am J Med Genet Part A 146: 930-3. [Crossref]

23. Brion M, Quintela I, Sobrino B, Torres M, Allegue C, et al. (2010) New technologies in the genetic approach to sudden cardiac death in the young. Forensic Sci Int 203: 15-24. [Crossref]

Copyright: (C2015 Osawa M. This is an open-access article distributed under the terms of the Creative Commons Attribution License, which permits unrestricted use, distribution, and reproduction in any medium, provided the original author and source are credited. 